\title{
Efeito protetor do nifurtimox contra a reativação parasitária em pacientes cronicamente infectados pelo Trypanosoma cruzi e tratados com corticóide em virtude de afecções associadas
}

\author{
Nifurtimox as a prophylatic drug to prevent reactivation in \\ chronic chagasic patients treated with corticoid \\ for associated diseases \\ Anis Rassi, Vicente Amato Neto, Astolpho Ferraz de Siqueiro \\ e Maurício Sérgio Brasil Leite
}

\begin{abstract}
Resumo Pacientes na fase crônica da doença de Chagas foram tratados com corticóide em virtude de afecções associadas e, a fim de tentar coibir reativação da infecção pelo Trypanosoma cruzi, houve uso concomitante do nifurtimox. Levando em conta o verificado em pesquisa anterior, quando corticóide de fato promoveu aumento da parasitemia detectada pelo xenodiagnóstico, pôde ser notado que o nifurtimox mostrou-se apto a evitar a citada acentuação parasitária, podendo tal constatação ser útil em procedimentos assistenciais, quando circunstancialmente estiverem presentes doença de Chagas e imunodepressão.
\end{abstract}

Palavras-chaves: Doença de Chagas. Fase crônica. Uso concomitante de corticóide e nifurtimox. Profilaxia de reativação parasitária.

\begin{abstract}
Patients in the chronic phase of Chagas' disease and receiving corticoid because of concommitant diseases were treated with nifurtimox. We proved in another paper that in the chronic phase of Chagas' disease corticoid use is associated with increased parasitemia, as evaluated by xenodiagnosis. In this study nifurtimox use prevented this increase, and we suggest that in immunocompromised patients with chronic Chagas' disease the use of this drug could be useful.
\end{abstract}

Key-words: Chagas' disease, chronic phase. Chagas' disease and corticoid use. Nifurtimox and corticoid use. Prophylaxis of Trypanosoma cruzi reactivation.

Departamento de Clínica Médica da Faculdade de Medicina da Universidade Federal de Goiás, Goiânia, GO. Endereço para correspondência: Prof. Anis Rassi. Av. A no 333, Setor Oeste, 74110-020 Goiânia, GO, Brasil. Tel: (062) 241-0035; Fax: (062) 281-8097 e 224-5886.

Recebido para publicação em 20/05/97. 
O binômio constituído por infecção pelo Trypanosoma cruzi e imunodepressão tem estado comumente em foco, merecendo considerações em estudos de várias ordens, que incluem também avaliações relacionadas com pacientes1 2467910 13. Contudo, é lícito reconhecer que ultimamente passou a tornar-se alvo de apreciações bem mais comuns porque déficit imunitário acontece com maior freqüência, como decorrência da influência de fatores de diferentes ordens, exemplificados pelo uso de determinados procedimentos terapêuticos, pela realização mais freqüente de transplante de órgãos e pela crescente participação do vírus da imunodeficiência humana (HIV), num contexto onde se encontram cerca de vinte milhões de indivíduos que estão infectados pelo parasito citado3 5.

Em pesquisa anterior, envolvendo enfermos tratados por meio de corticóide, em virtude de afecções associadas à doença de Chagas, foi demonstrado evidente aumento da parasitemia por meio do xenodiagnóstico12. Tal fato pôde ser valorizado em comparação com o verificado no grupo controle. Como é absolutamente necessário proceder satisfatoriamente em termos assistenciais, procurando eliminar ou atenuar a ação parasitária, vale a pena investigar se a administração de tripanosomicida auxilia no sentido de opor-se ao aumento da quantidade do agente causal e de prevenir reativação do processo infeccioso nos imunodeprimidos 29 .

Levando em conta o propósito citado, a doentes que por motivos diversos precisaram receber corticóide, administramos concomitantemente o nifurtimox, antiparasitário dotado de comprovada ação sobre o T. cruzi. Nesta comunicação relatamos o que comprovamos a respeito, com o intuito de contrabalançar a exacerbação do parasitismo que a corticoidoterapia é capaz de promover.

\section{MATERIAL E MÉTODOS}

Compuseram a casuística 10 pacientes na fase crônica da doença de Chagas, 7 sem cardiopatia detectável eletrocardiográfica e radiologicamente e 3 com cardiopatia incipiente. A idade variou entre 18 e 53 anos (média de 40,5 anos $\pm 14,4$ ), sendo 5 do sexo masculino. O diagnóstico da parasitose decorreu de prova sorológica de fixação do complemento. As afecções associadas que motivaram o emprego de corticóide (triancinolona em 6 pacientes e prednisona em 4) foram: pênfigo foliáceo em 6 casos, púrpura trombocitopênica em 3 e doença de Hodgkin em um. Os doentes permaneceram internados, durante a etapa de administração do corticóide e do nifurtimox, no Hospital do Pênfigo (atual Hospital de Doenças Tropicais Dr. Anuar Auad) ou no Hospital do Câncer (atual Hospital Araújo Jorge), ambos sediados em Goiânia, GO.

As doses diárias do corticóide, expressas em prednisona, variaram de $80 \mathrm{mg}$ (início do tratamento) a 5,8mg (final do tratamento), com administração progressivamente decrescente, de 12 em 12 horas, via oral, por período de 60 dias, à exceção de um caso, cuja duração foi de 52 dias. Calculadas como médias, foram de $52 \mathrm{mg}$ por dia entre o $1^{\circ}$ e o $15^{\circ}$ dias de tratamento, de $40 \mathrm{mg}$ entre o $16^{\circ}$ e o $30^{\circ}$, de $35 \mathrm{mg}$ entre o $31^{\circ}$ e o $45^{\circ}$ e de $30 \mathrm{mg}$ entre o 46을 e o 60․

O nifurtimox foi empregado concomitantemente, por via oral, de $8 \mathrm{em} 8$ horas, na dose de $10 \mathrm{mg} / \mathrm{kg}$ de peso/dia durante 60 dias, em média, à exceção de 2 casos, em que sua administração foi interrompida (aos 23 e 26 dias), em virtude de efeito colateral (distúrbio do comportamento).

Das prescrições também fizeram parte ciclofosfamida no caso de Hodgkin, antibiótico (tetraciclina, usada em 6 pacientes) e outras drogas, indicadas para atenuar ou debelar efeitos colaterais.

Antes de ser iniciado o emprego de corticóide e nifurtimox foram realizados xenodiagnóstico, inoculação de sangue em camundongos, xenodiagnóstico nos animais, provas de precipitina e de fixação do complemento com soro sangüíneo, eletrocardiograma e avaliação radiológica da área cardíaca. Repetimos estes exames em geral 4 vezes, de 15 em 15 dias, aproximadamente, no decurso da utilização das drogas, para obter a dedução almejada. $\mathrm{Na}$ Tabela 1, estão enumeradas as vezes em que praticamos estes controles, depois de iniciada a terapêutica.

Sobre os exames em questão, efetivados paralelamente a reiterado acompanhamento clínico, indicamos a seguir informes referentes às técnicas utilizadas.

Xenodiagnóstico: com 20 ninfas de terceiro ou quarto estádios do Triatoma infestans; análise das fezes, individualmente, cerca de 45 dias depois do repasto, com sacrifício dos insetos.

Inoculação do sangue em camundongo: $1 \mathrm{ml}$ com citrato de sódio a $3 \%$ no peritônio; sacrifício após 15 a 20 dias, havendo prévio 
xenodiagnóstico com cinco ninfas de terceiro ou quarto estádios do $T$. infestans e análise das fezes, individualmente, cerca de 45 dias depois do repasto, com sacrifício dos insentos; em músculos cardíaco e esquelético do roedor houve procura, histologicamente, de formas amastigotas do $T$. cruzi após coloração pela hematoxilina-eosina.

Prova sorológica da precipitina: segundo Muniz e Freitas8, ela é comumente positiva na fase aguda da doença de Chagas e, incluindoa, pretendíamos evidenciar eventual reativação.

Prova sorológica de fixação do complemento: conforme processo descrito por Pedreira de Freitas e Almeida11.

Eletrocardiograma e avaliação radiológica da área cardíaca: de acordo com as consagradas e habituais maneiras de executálos.

Tabela 1 - Exames efetuados como controles e números de vezes em que foram realizados.

\begin{tabular}{|c|c|c|c|c|c|c|c|}
\hline Caso & $X D$ & $1 \mathrm{C}^{*}$ & $\mathrm{XDC}^{*}$ & PP & RFC & ECG & ERAC \\
\hline 1 & 4 & 1 & 1 & 4 & 4 & 4 & 4 \\
\hline 2 & 4 & 3 & 3 & 4 & 4 & 4 & 4 \\
\hline 3 & 4 & 2 & 2 & 4 & 4 & 4 & 4 \\
\hline 4 & 4 & 3 & 3 & 3 & 4 & 4 & 4 \\
\hline 5 & 4 & 2 & 2 & 4 & 4 & 4 & 4 \\
\hline 6 & 4 & 4 & 4 & 4 & 4 & 4 & 4 \\
\hline 7 & 4 & 1 & 1 & 4 & 3 & 4 & 4 \\
\hline 8 & 4 & 3 & 3 & 4 & 4 & 4 & 4 \\
\hline 9 & 4 & 3 & 3 & 3 & 4 & 4 & 4 \\
\hline 10 & 3 & 3 & 2 & 2 & 3 & 3 & 3 \\
\hline
\end{tabular}

$\mathrm{XD}=$ xenodiagnóstico; $\mathrm{IC}$ = inoculação de sangue em camundongo; $\mathrm{XDC}=$ xenodiagnóstico em camundongo; PP = prova de precipitina; $\mathrm{RFC}$ = reação de fixação do complemento; ECG = eletrocardiograma; ERAC = exame radiológico da área cardíaca.

* Nos casos 3 e 7 foi usado um camundongo em cada inoculação, mas nos demais dois ou três compuseram as diferentes provas.

\section{RESULTADOS}

Nunca houve evidenciação do T. cruzi às inoculações, dizendo respeito estas negatividades à procura nos músculos cardíaco e esquelético, como ainda por intermédio dos xenodiagnósticos efetuados nos animais.

A prova de fixação do complemento evidenciou resultados muito heterogêneos nos diferentes casos, traduzidos por reagentes (com títulos diagnósticos e não-diagnósticos) e nãoreagentes. Comportamento semelhante teve o teste da precipitina, que evidenciou muitas negatividades, esporadicamente surgindo resultados positivos, porém, sem vínculos específicos com determinados pacientes ou situações.

O acompanhamento clínico e as avaliações radiológico-eletrocardiográficas não demonstraram mudanças referentes ao quadro clínico da doença de Chagas.

Os 10 xenodiagnósticos realizados antes do tratamento envolveram 200 triatomíneos e detectaram o T. cruzi em 4 casos, estando 39 insetos infectados entre os 198 vivos examinados. Por sua vez, considerando os 39 (em uma paciente foram feitos 3 e não 4 exames) praticados durante o tratamento, com a utilização de 780 triatomíneos, obtivemos positividade em um só caso, estando apenas 4 insetos infectados entre 735 vivos examinados (um aos 15 dias e 3 aos 45 dias de tratamento). Estes resultados estão representados na Figura 1, correlacionados com a média das doses diárias de corticóide.

Desta investigação fizeram parte 2 pacientes que, em ensaio anterior (Rassi e cols, 1997), haviam recebido corticóide (caso de pênfigo foliáceo) e corticóide associado a ciclofosfamida (caso de Hodgkin), e que, por terem apresentado recorrência do quadro clínico meses após, aqui foram incluídos, repetindo, praticamente, o mesmo esquema terapêutico anterior, agora acrescido de nifurtimox. As Figuras 2 e 3 mostram o comportamento do xenodiagnóstico nos 2 casos e em ambas as situações.

Efeitos colaterais foram observados, isolada ou associadamente, em 9 casos, assim distribuídos: hiporexia em 8 pacientes, emagrecimento (perda de cerca de $10 \%$ do peso corpóreo) em 5 , náuseas e/ou vômitos em 5 , insônia em 4 , distúrbio do comportamento 


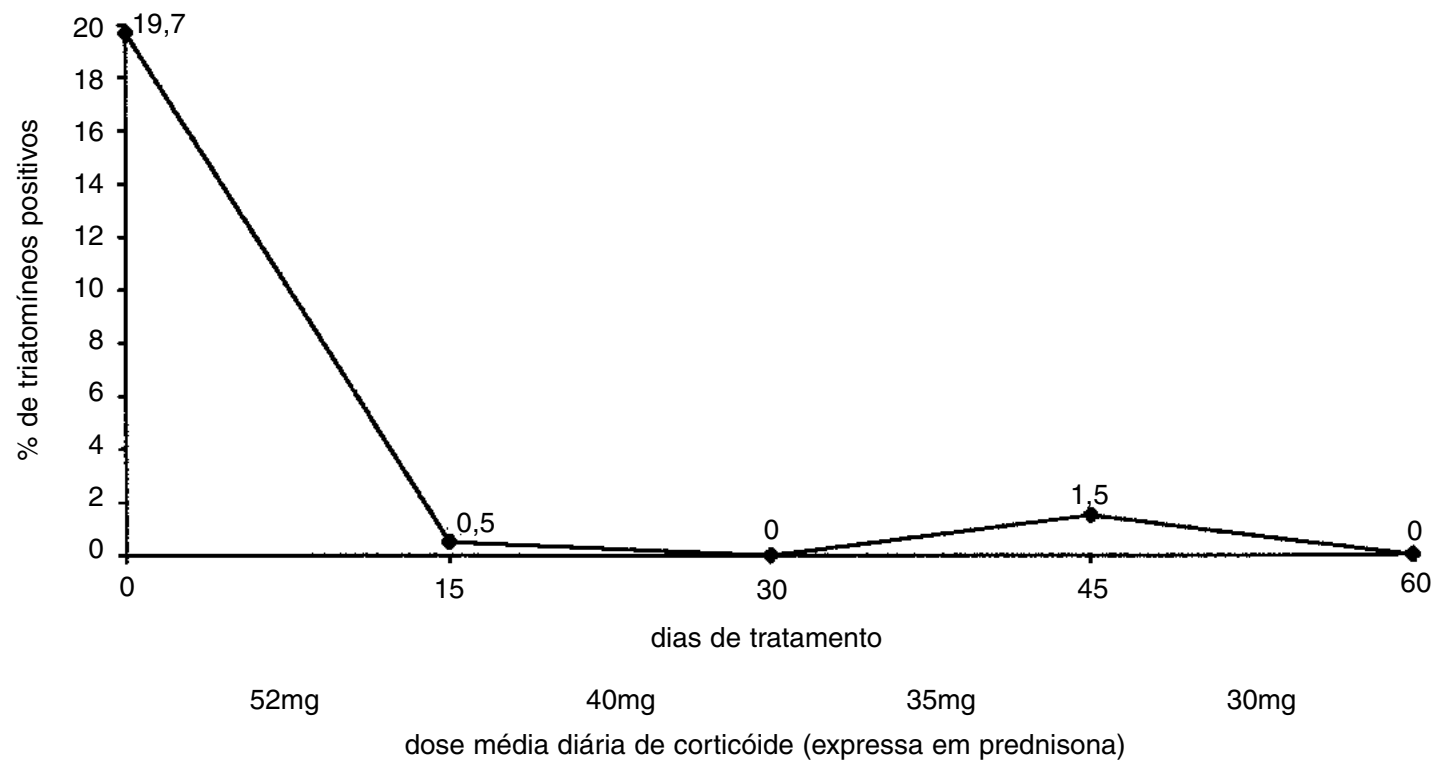

Figura 1 - Positividade de triatomíneos, ao xenodiagnóstico, em 10 pacientes submetidos a tratamento com corticóide associado ao nifurtimox. Observa-se expressiva redução da positividade, que chega o zero aos 60 dias de tratamento.

em 3, parestesia nas extremidades dos membros em 2, tremores nas extremidades dos membros em 2 e dermopatia alérgica em um.
Foi-nos possível acompanhar 3 dos 10 pacientes da série; em todos eles o xenodiagnóstico resultou positivo (1ํㅜ $2^{\circ}$ e $6^{\circ}$ meses após o tratamento).

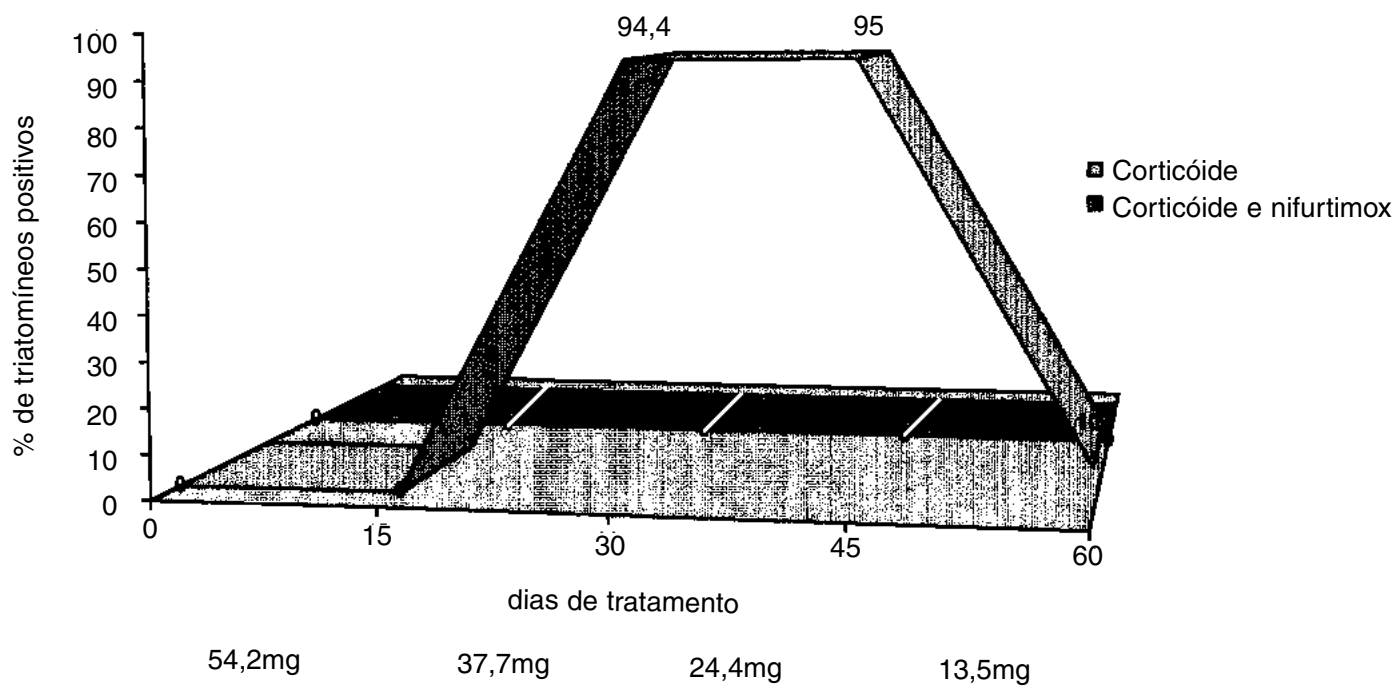

dose média diária de corticóide - betametasona (expressa em prednisona)

Figura 2 - Positividade de triatomíneos, ao xenodiagnóstico, em paciente tratada com corticóide e, 9 meses após, com corticóide e nifurtimox. Durante o uso de corticóide houve acentuado aumento do percentual de triatomíneos positivos, relacionado com a dose do medicamento, fato não observado quando ao corticóide foi associado o nifurtimox, etapa em que o percentual de triatomíneos positivos foi sempre de zero, o que demonstra, no mínimo, importante atividade supressiva do tripanosomicida. 


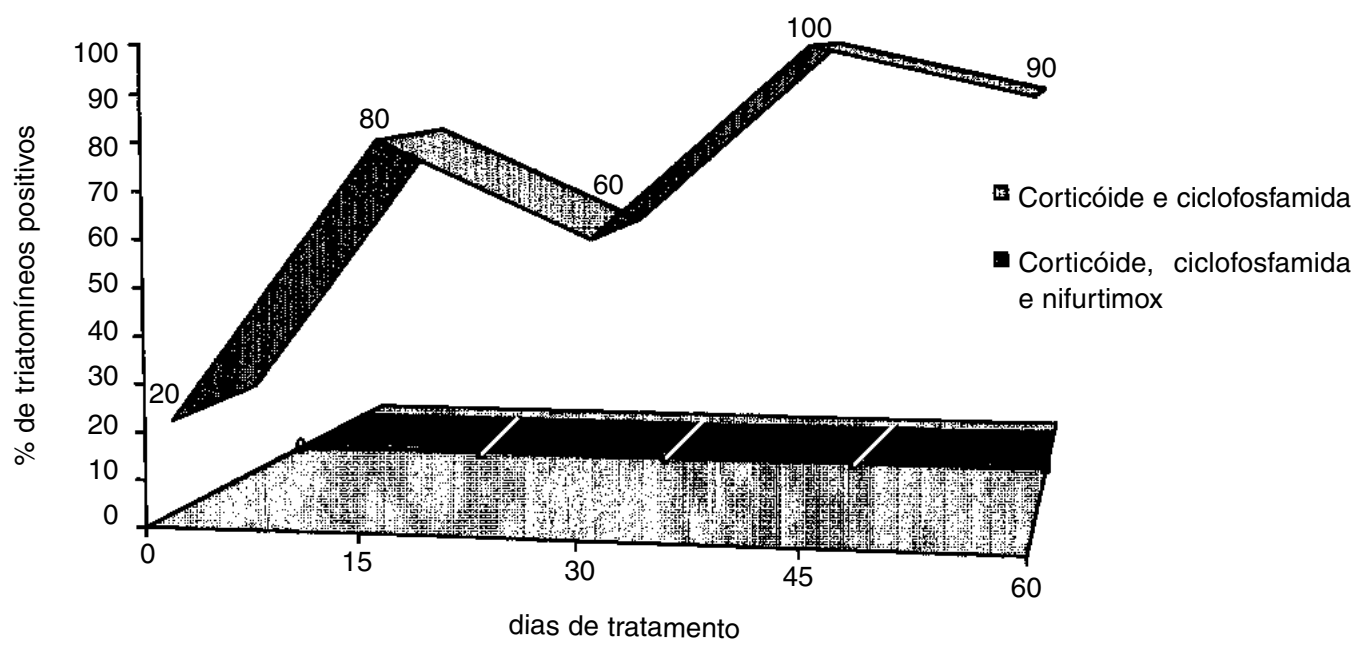

$40 \mathrm{mg} \quad 40 \mathrm{mg} \quad 40 \mathrm{mg} \quad 40 \mathrm{mg}$

dose média diária de corticóide (prednisona)

Figura 3 - Positividade de triatomíneos, ao xenodiagnóstico, em paciente tratado com corticóide e ciclofosfamida e, 11 meses após, com corticóide, ciclofosfamida e nifurtimox. No primeiro tratamento ocorreu acentuado aumento do percentual de triatomíneos positivos, fato não observado no segundo, no qual o percentual foi sempre de zero, o que demonstra, no mínimo, importante atividade supressiva do nifurtimox. Dose da ciclofosfamida: 200mg/dia, por via endovenosa, durante 44 dias no primeiro tratamento e 24 dias no segundo.

\section{DISCUSSÃO}

Diante dos resultados expostos, percebe-se que para fazer julgamento sobre o que houve com a intensidade parasitária só pudemos contar com os informes derivados do xenodiagnóstico, no conjunto de providências que adotamos com esta finalidade. Inoculação em camundongo, fixação do complemento e precipitina não ajudaram, em virtude de negatividades sistemáticas e do comportamento extremamente heterogêneo notado à evidenciação de anticorpos, respectivamente. No tocante ao comportamento da fixação do complemento, não cremos que para tanto tenha influído o nifurtimox, pois, não é esta a nossa experiência. Interferência do corticóide na anticorpogênese ou variações temporais espontâneas são explicações cogitáveis que, sem elementos cabais, não nos permitimos endossar.

Quanto à doença de Chagas, as avaliações clínica, eletrocardiográfica e radiológica não evidenciaram alterações dignas de registro, indicando que o binômio corticóide-nifurtimox, no período de investigação considerado, não influiu em seu quadro.
A fim de obtermos dedução relacionada com o propósito do estudo valorizamos os resultados do xenodiagnóstico e dedução de pesquisa realizada por Rassi e cols12, que analisaram o sucedido em casuística parecida com a da análise que agora comunicamos, quando procuraram conhecer a interferência de corticóide na intensidade parasitária de indivíduos com doença de Chagas.

Com efeito, Rassi e cols 12 concluíram que, indiscutivelmente, o corticóide conduziu a aumento do número de tripanosomos no sangue, ou seja, exacerbou a parasitemia, demarcada pelo xenodiagnóstico, em plano avaliador amplo que que revelou a precária cooperação de vários outros recursos adotados. Ao mesmo tempo, notaram que maiores elevações parasitêmicas podiam ser devido às mais elevadas doses de corticóide.

Cremos que dois argumentos indicam que o emprego do nifurtimox atenua a acentuação da parasitemia promovida pela terapia com corticóide. O primeiro é a percepção de que, nos exames de controle, o número de triatomíneos 
infectados apareceu como acentuadamente menor do que o verificado nos xenodiagnósticos iniciais, apesar dos exames terem sido mais numerosos. Especificando, lembramos que em 10 executados antes do uso concomitante de corticóide e nifurtimox registramos 39 insetos positivos num total de 198 vivos, enquanto que, no seguimento, apenas 4 insetos se mostraram infectados entre 735 vivos examinados. Portanto, mesmo em procuras bem mais numerosas encontramos quantidade nitidamente menor de triatomíneos albergando o T. cruzi. O outro parâmetro que nos serviu para comparação advém do trabalho de Rassi e cols12, que observaram evidente exacerbação da parasitemia quando só o corticóide foi usado. Vale referir ainda, a observação exposta nas Figuras 2 e 3.

Em 2 casos o período de uso do nifurtimox foi bem menor do que o do corticóide. Isso aconteceu porque o antiparasitário provocou efeito adverso que impediu o prosseguimento de sua administração. É importante frisar que os 4 triatomíneos positivos, nos exames de controle, foram encontrados em um destes 2 casos de utilização mais curta do fármaco antiparasitário.

Assim, há que admitir-se que o nifurtimox coibiu a elevação parasitária e que dedução deste tipo respalda a adoção de conduta quando, como decorrência de imunodepressão, ficar temido o agravamento da infecção pelo T. cruzi, capaz de causar graves comprometimentos orgânicos.

No momento, infelizmente, só contamos com os préstimos do nifurtimox e do benznidazol como agentes terapêuticos etiológicos quanto à doença de Chagas. Eles não são dotados da eficácia desejada e podem desencadear manifestações colaterais de graus variáveis, principalmente o nifurtimox. Todavia, mesmo assim, podem ser úteis, enquanto esperamos o advento de novos medicamentos.

Em comunicação posterior relataremos o que propiciou o benznidazol em estudo congênere a este que agora estamos divulgando.

\section{AGRADECIMENTOS}

Os autores são gratos à Professora Simonne A. Silva, do Instituto de Patologia Tropical e Saúde Pública da Universidade Federal de Goiás, pela elaboração das curvas de positividade dos triatomíneos ao xenodiagnóstico.

\section{REFERÊNCIAS BIBLIOGRÁFICAS}

1. Abath FGC, Gomes YM, Coutinho EM, Montenegro $S M L$, Melo MEB, Carvalho AB. Effects of betamethasone on the course of experimental infection with Trypanosoma cruzi. Revista da Sociedade Brasileira de Medicina Tropical 19:161164, 1986.

2. Andrade SG, Andrade ZA, Sadigursky M. Combined treatment with a nitrofuranic and a corticoid in experimental Chagas' disease in the dog. American Journal of Tropical Medicine and Hygiene 29:766773, 1980.

3. Bocchi EA. Transplante cardíaco em portadores da cardiopatia chagásica. Revista da Sociedade de Cardiologia do Estado de São Paulo 4:198-204, 1994.

4. Brener Z, Chiari E. The effects of some immunosuppressive agents in experimental chronic Chagas's disease. Transactions of the Royal Society of Tropical Medicine and Hygiene 65:629-636, 1971.

5. Chocair PR, Amato Neto V, Sabbaga E, Torecillas $\mathrm{PH}$. Aspectos clínico-diagnósticos relativos à fase aguda da doença de Chagas, em pacientes submetidos à transplante de rim e imunodeprimidos. Revista da Sociedade Brasileira de Medicina Tropical 18:43-45, 1985.

6. França LCM, Fleury RN, Ramos Jr HA, Lemos S, Melaragno Filho R, Pasternak J. Moléstia de Chagas crônica associada a leucemia linfática: ocorrência de encefalite aguda como alteração do estado imunitário. Arquivos de Neuro-Psiquiatria 27:59-66, 1969.

7. Kohl S, Pickering LK, Frankel LS, Yaeger RG. Reactivation of Chagas' disease during therapy of acute lymphocytic leukemia. Cancer 50:827-828, 1982.

8. Muniz J, Freitas G. Contribuição para o diagnóstico da doença de Chagas pelas reações de imunidade. II - Isolamento de polissacarídeos de Schizotrypanum cruzi e de outros Tripanosomídeos, seu comportamento nas reações de precipitação, de fixação do complemento e de hipersensibilidade. Os "tests" de floculação (sublimado e formol-gel). Revista Brasileira de Biologia 4:421-438, 1944.

9. Okumura M, Amato Neto V, Kitagawa MM, Takamatsu NK, Ishikawa PS, lizuza FH, Iriya K. Atividade terapêutica do benzonidazol em camundongos infectados pelo Trypanosoma cruzi e imunodeprimidos por associação de ciclosporina com prednisona. Revista do Hospital das Clínicas da 
Faculdade de Medicina da Universidade de São Paulo 45:260-262, 1990.

10. Okumura M, Décourt LV. Estudo de efeitos da administração de drogas imunodepressoras sôbre a moléstia de Chagas experimental. Revista do Hospital das Clínicas da Faculdade de Medicina da Universidade de São Paulo 24:335-342, 1969.

11. Pedreira de Freitas JL, Almeida JO. Nova técnica de fixação do complemento para moléstia de Chagas (Reação quantitativa com antígeno gelificado de culturas de Trypanosoma cruzı). O Hospital 35:787800, 1949.
12. Rassi A, Amato Neto V, Siqueira AF, Doles J, Leite MSB, Silva OQ, Cardoso VM. Influência de corticóide, na doença de Chagas crônica, administrado em virtude de afecções associadas. Revista da Sociedade Brasileira de Medicina Tropical 30:93-99, 1997.

13. Vichi FL, Albuquerque RD, Santos J, Soubihe NV, Moreira AC, Gil Netto J. The effect of oral treatment with prednisone on T. cruzi parasitemia of patients with chronic Chagas' heart disease. Revista do Instituto de Medicina Tropical de São Paulo 11:280284, 1969. 